\title{
Search for self-interacting dark matter and other dark sector searches with the BABAR detector
}

\author{
Gerald Eigen \\ * and On behalf of the $B A B A R$ Collaboration \\ University of Bergen, \\ Allegaten 55, 5007 Bergen, Norway \\ E-mail: gerald.eigen@uib.no
}

\begin{abstract}
A new class of dark matter models introduces a dark sector with new Dirac fermions charged under an additional U(1) gauge group. The corresponding gauge boson, the dark photon $A^{\prime}$, has an $\mathrm{MeV} / c^{2}-\mathrm{GeV} / c^{2}$ mass and couples to the Standard Model photon (and $\mathrm{Z}$ ) via kinetic mixing. Fermionic bound states ("darkonium" $\Upsilon_{\mathrm{D}}$ ) could form if the dark sector coupling constant is strong enough. We present a search for dark sector bound states $\left(\Upsilon_{\mathrm{D}}\right)$ in $e^{+} e^{-} \rightarrow \gamma \Upsilon_{\mathrm{D}}, \Upsilon_{\mathrm{D}} \rightarrow A^{\prime} A^{\prime} A^{\prime}$, $A^{\prime} \rightarrow X^{+} X^{-}(X=e, \mu, \pi)$ decays for $0.02 \mathrm{GeV} / c^{2} \leq m_{\mathrm{A}^{\prime}} \leq 1.0 \mathrm{GeV} / c^{2}, 1 \mathrm{GeV} / c^{2} \leq m_{\Upsilon_{\mathrm{D}}} \leq 10$ $\mathrm{GeV} / c^{2}$ using the full data sample collected with the $B A B A R$ detector. Furthermore, axion-like particle and leptophilic dark matter may be produced in $e^{+} e^{-}$collisions.
\end{abstract}

\footnotetext{
*** The European Physical Society Conference on High Energy Physics (EPS-HEP2021), *** *** 26-30 July $2021 * * *$

*** Online conference, jointly organized by Universit\$t Hamburg and the research center DESY ***
}

\footnotetext{
${ }^{*}$ Speaker
} 


\section{Introduction}

The Standard Model (SM) may be connected to the dark sector through so-called portals. These links are the lowest-dimensional operators that may provide coupling of the dark sector to the SM as higher-dimensional operators are mass suppressed [1,2]. They include the vector portal, the scalar portal, the neutrino portal and the axion-like portal. The vector portal contribution is $\mathcal{L}_{\text {vector }}=\epsilon F^{\mu \nu} F_{\mu \nu}^{\prime}$ where $F^{\mu \nu}$ and $F_{\mu \nu}^{\prime}$ are the electromagnetic tensor and the dark photon tensor, respectively, where the latter is introduced by a new $\mathrm{U}(1)$ symmetry. The parameter $\epsilon$ denotes the kinetic mixing between the dark photon and the SM [3,4]. The scalar portal contribution is $H^{2}\left(\mu \phi+\lambda \phi^{2}\right)$, where $H$ is the Higgs field and $\phi$ is the dark scalar field. In addition, we have the electroweak parameters $\mu$ and $\lambda$. This portal introduces a new dark scalar that mixes with the Higgs field. The neutrino portal involves a new heavy neutral lepton that mixes with the left-handed SM doublet and Higgs boson. A new axion or axion-like particle introduces a new term $f_{\mathrm{a}}^{-1} F^{\mu \nu} F_{\mu \nu} a$, where $a$ is the axion field and $f_{\mathrm{a}}$ is a coupling. The new axion or axion-like particle couples to SM gauge and fermion fields. Here, we focus on a search for self-interacting dark matter and briefly summarize our results on searches for axion-like particles and leptophilic dark matter.

\section{Search for Self-interacting Dark Matter (Darkonium)}

A dark photon $A^{\prime}$ may couple to a dark fermion anti-fermion pair $(\chi \bar{\chi})$. If the $A^{\prime}-\chi$ coupling constant $\alpha_{\mathrm{D}}$ is large enough, the $\chi \bar{\chi}$ pair could form a bound state, called darkonium [5, 6]. This represents self-interacting dark matter. The lowest states are $J^{\mathrm{PC}}=0^{-+}$(pseudoscalar) and $J^{\mathrm{PC}}=1^{--}$(vector). In analogy with the SM they are called $\eta_{\mathrm{D}}$ and $\Upsilon_{\mathrm{D}}$. These states may be produced in $e^{+} e^{-} \rightarrow A^{\prime} \eta_{\mathrm{D}}, \eta_{\mathrm{D}} \rightarrow A^{\prime} A^{\prime}$ and $e^{+} e^{-} \rightarrow \gamma \Upsilon_{D}, \Upsilon_{\mathrm{D}} \rightarrow A^{\prime} A^{\prime} A^{\prime}$, depicted in Fig. 1 (left). Using data collected at the $\Upsilon(4 S), \Upsilon(3 S), \Upsilon(2 S)$ and off-resonance corresponding to an integrated luminosity of $514 \mathrm{fb}^{-1}, B A B A R$ has searched for the $\Upsilon_{\mathrm{D}}$ in which the dark photons decayed to $e^{+} e^{-}$, $\mu^{+} \mu^{-}$or $\pi^{+} \pi^{-}$[7]. Both prompt and delayed decays were considered.
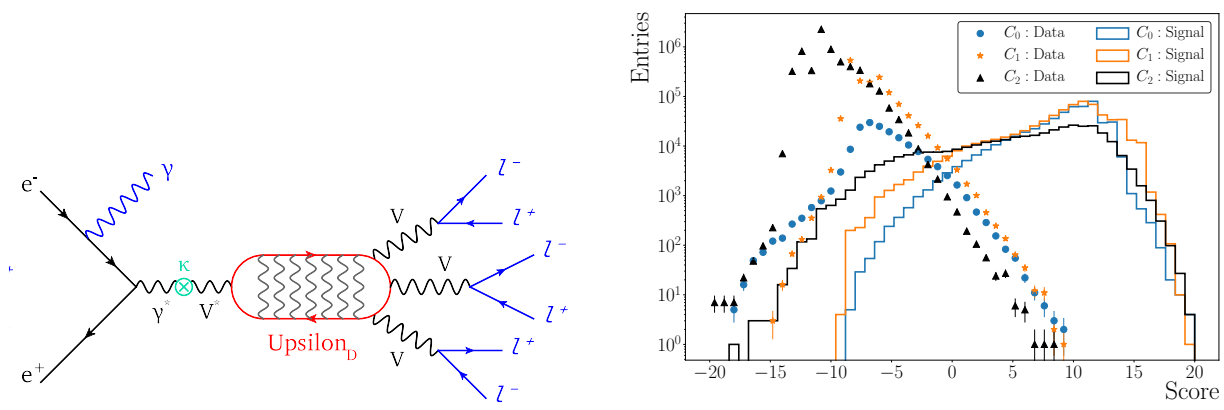

Figure 1: Left: Feynman diagram for $\Upsilon_{D}$ production and decay. Right: Distributions of the random forrest classifier scores for each event category for the data (markers) and signal Monte Carlo (solid lines) samples.

In $B A B A R$, we select six charged tracks with at most two $\pi^{+} \pi^{-}$pairs to reduce the large QCD background. The event has to be consistent with an ISR process. Excluding the ISR photon the remaining neutral energy in the calorimeter has to be small and the masses of the three dark photons have to be similar. For prompt decays, we consider dark photon masses of 
$0.001<m_{\mathrm{A}^{\prime}}<3.16 \mathrm{GeV} / c^{2}$ and darkonium masses of $0.05<m_{\Upsilon_{\mathrm{D}}}<9.5 \mathrm{GeV} / c^{2}$. We have generated 119 different $A^{\prime}$ and $\Upsilon_{\mathrm{D}}$ mass hypotheses. For dark photon masses below $0.20 \mathrm{GeV} / c^{2}$, we consider flight paths of $c \tau=0.1,1$ and $10 \mathrm{~mm}$. We classify the events into three classes with respect to the number of $\pi^{+} \pi^{-}$pairs and train three multivariate classifiers consisting of logistic regressions stacked on top of Random Forest classifiers, which are trained using 13 input variables. We transform the classifier outputs into classifier scores using a logit function [8]. Higher scores indicate higher consistency with being a signal event. Figure 1 (right) shows the random forrest classifier scores in comparison to data. We combine events of all categories into a single sample and maximize a figure of merit averaged over a wide range of $\Upsilon_{\mathrm{D}}$ and $A^{\prime}$ masses to obtain the optimal selection. We define the $\left(m_{\Upsilon_{\mathrm{D}}}, m_{A^{\prime}}\right)$ signal region as $\left(m_{\Upsilon_{\mathrm{D}}}-4 \sigma_{m_{\Upsilon_{\mathrm{D}}}}, m_{\Upsilon_{\mathrm{D}}}+4 \sigma_{m_{\Upsilon_{\mathrm{D}}}}\right)$ and $\left(m_{A^{\prime}}-4 \sigma_{m_{A^{\prime}}}, m_{A^{\prime}}+4 \sigma_{m_{A^{\prime}}}\right)$. The background is estimated in the sideband regions $\left(m_{\Upsilon_{\mathrm{D}}}-\right.$ $\left.8 \sigma_{m_{\Upsilon_{\mathrm{D}}}}, m_{\Upsilon_{\mathrm{D}}}-4 \sigma_{m_{\Upsilon_{\mathrm{D}}}}\right)$ and $\left(m_{\Upsilon_{\mathrm{D}}}+4 \sigma_{m_{\Upsilon_{\mathrm{D}}}}, m_{\Upsilon_{\mathrm{D}}}+8 \sigma_{m_{\Upsilon_{\mathrm{D}}}}\right)$. The mass resolutions are 1-8 MeV/c ${ }^{2}$ for the dark photon and 5-40 MeV/c for $\Upsilon_{\mathrm{D}}$.

After applying all selection criteria we see 69 signal events, where at most two events fall into one signal bin. Figure 2 (left) shows the distribution of observed events in the $\left(m_{\Upsilon_{\mathrm{D}}}, m_{A^{\prime}}\right)$ plane. The distribution is consistent with the expected background. Thus, we derive $90 \%$ confidence level (CL) upper limits on the $e^{+} e^{-} \rightarrow \gamma \Upsilon_{\mathrm{D}}$ cross section using a profile likelihood method. Enhancements near $0.8 \mathrm{GeV} / c^{2}$ in $m\left(\pi^{+} \pi^{-}\right)$and $1.0 \mathrm{GeV} / c^{2}$ in $m\left(K^{+} K^{-}\right)$come from $\rho$ and $\phi$ decay backgrounds. In all other regions we see no enhancements yielding $90 \% \mathrm{CL}$ cross section upper limits of $10^{-2}$ to $1 \mathrm{fb}^{-1}$.
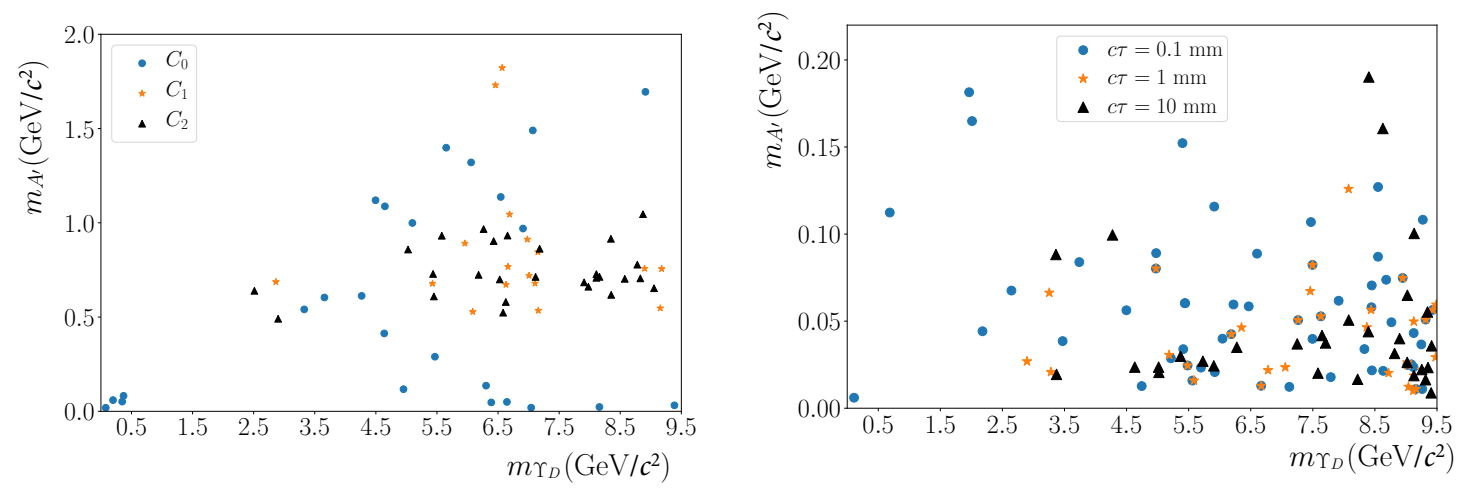

Figure 2: Left: Signal candidates for prompt dark photon decays in the $\left(m_{\Upsilon_{\mathrm{D}}}, m_{A^{\prime}}\right)$ plane. Right: Signal candidates for dark photons with three different lifetime hypotheses in the $\left(m_{\Upsilon_{\mathrm{D}}}, m_{A^{\prime}}\right)$ plane.

For low-mass dark photons, the lifetime may become longer. Thus we search for events with decay vertices of $c \tau=0.1,1$ and $10 \mathrm{~mm}$. To determine cross sections for the three lifetime hypotheses, we pursue a similar procedure as before and train a classifier for each $c \tau$. The selection yields 56, 33 and 31 signal candidates, respectively, which are consistent with the expected background. The $90 \%$ confidence level upper limits on the $e^{+} e^{-} \rightarrow \gamma \Upsilon_{\mathrm{D}}$ cross section are about six orders of magnitude lower than those for prompt decay.

Since we see no signals, we extract $90 \%$ confidence level upper limits on the kinetic mixing parameter $\epsilon$ in an iterative procedure taking the lifetime into account. We derive separate upper limits as a function of $m_{\mathrm{A}^{\prime}}$ for several values of the dark sector coupling constant $\alpha_{\mathrm{D}}$ and for different 

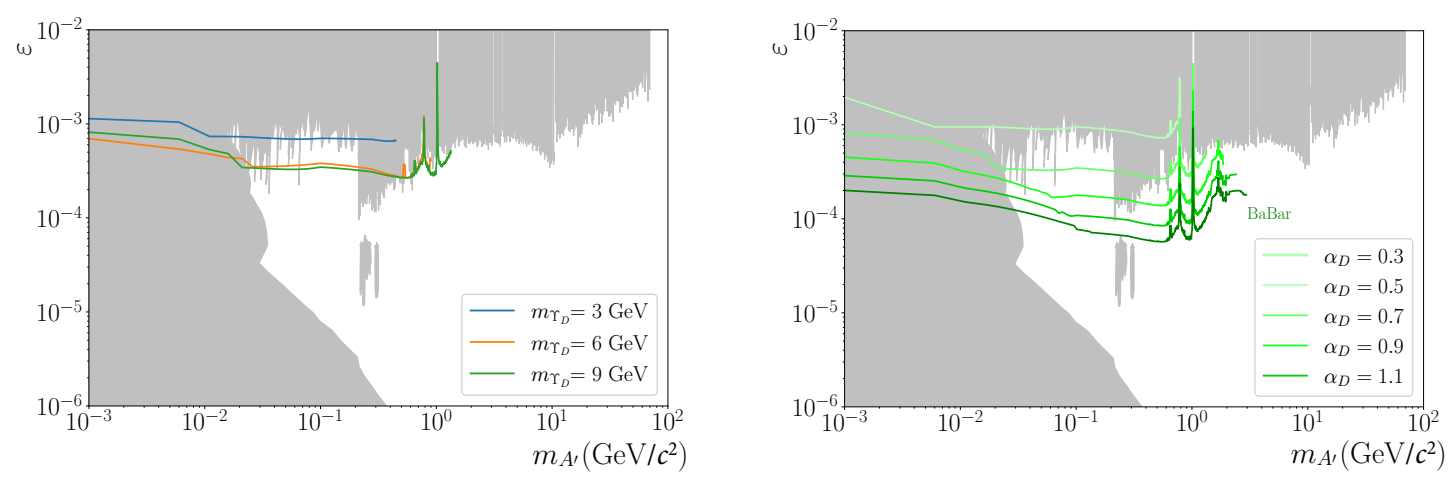

Figure 3: Left: The $90 \%$ confidence level upper limits on $\epsilon$ as a function of $m_{\mathrm{A}^{\prime}}$ for $m_{\Upsilon_{\mathrm{D}}}=3,6,9 \mathrm{GeV} / c^{2}$ and $\alpha_{\mathrm{D}}=0.5$. Right: The $90 \%$ confidence level upper limits on $\epsilon$ as a function of $m_{\mathrm{A}^{\prime}}$ for five values of $\alpha_{\mathrm{D}}=0.3,0.5,0.7,0.9$, and 1.1 for $m_{\Upsilon_{\mathrm{D}}}=9 \mathrm{GeV} / c^{2}$.

darkonium masses. Figure 3 (left) shows the BABAR 90\% confidence level upper limits on $\epsilon$ versus the dark photon mass for three values of $m_{\Upsilon_{D}}$. Our measurements improve upon existing constraints over a significant fraction of dark photon masses below $1 \mathrm{GeV} / \mathrm{c}^{2}$. For a large fraction of parameter space we set bounds on $\epsilon$ of $5 \times 10^{-5}-10^{-3}$. Bounds improve with higher dark photon masses, larger darkonium masses and larger values of $\alpha_{\mathrm{D}}$. Since these upper limits are still statistics limited, Belle II will eventually produce improved upper limits.

\section{Search for Axion-like Particles}

Axion-like particles (ALP) are new light pseudoscalars that couple dominantly to gauge bosons. In the presence of couplings to $\mathrm{SU}(2)$ gauge bosons we may get a large flavor-changing neutralcurrent (FCNC) production rate since ALP production is at a lower order in effective field theory than the corresponding Standard Model FCNC processes. At small mass and coupling the lifetime may be large yielding decay lengths of the order of millimeters to tens of centimeters. BABAR has searched for ALPs in the decay $B \rightarrow K a, a \rightarrow \gamma \gamma$ [9]. To form $B$ candidates, we combine a well-identified kaon with two photons and apply a kinematic fit to improve axion mass resolution. Then, we train two boosted decision trees to separate signal from $e^{+} e^{-} \rightarrow B \bar{B}$ and $e^{+} e^{-} \rightarrow q \bar{q}$ backgrounds $(q=u, d, s, c)$, respectively. The $m_{\gamma \gamma}$ invariant-mass spectrum shows peaking backgrounds at the $\pi^{0}, \eta$ and $\eta^{\prime}$ masses that we exclude. We extract the signal as a function of axion mass with fits over sliding mass intervals. We see no significant signals and set $90 \% \mathrm{CL}$ upper limits on the branching fraction $B \rightarrow K a, a \rightarrow \gamma \gamma$ for prompt and displaced decays as shown in Figs. 4 (left, right). For prompt decays all upper limits are below $10^{-6}$. For displaced vertices, the upper limits increase with $c \tau$ in particular for low ALP masses. We further set $90 \%$ CL upper limits on the $a-W$ coupling parameter $g_{\mathrm{aW}}$ as a function of the ALP mass depicted in Fig. 5 (left). Over a wide mass range the $90 \%$ upper limits are improved by over two orders of magnitude. 

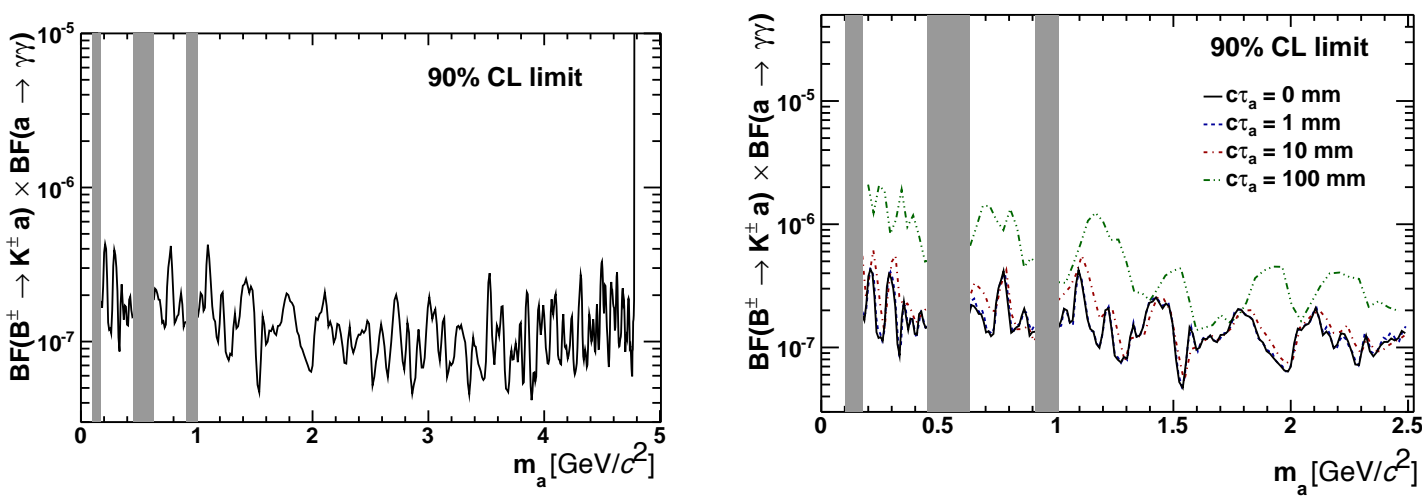

Figure 4: The 90\% CL upper limits on the branching fraction $B \rightarrow K a, a \rightarrow \gamma \gamma$ for promptly decaying ALPs (left) and for decay length between 0 and $100 \mathrm{~mm}$ for $m_{\mathrm{a}}<2.5 \mathrm{GeV} / c^{2}$ (right). The grey bars show the $\pi^{0}, \eta$ and $\eta^{\prime}$ regions that have been excluded.
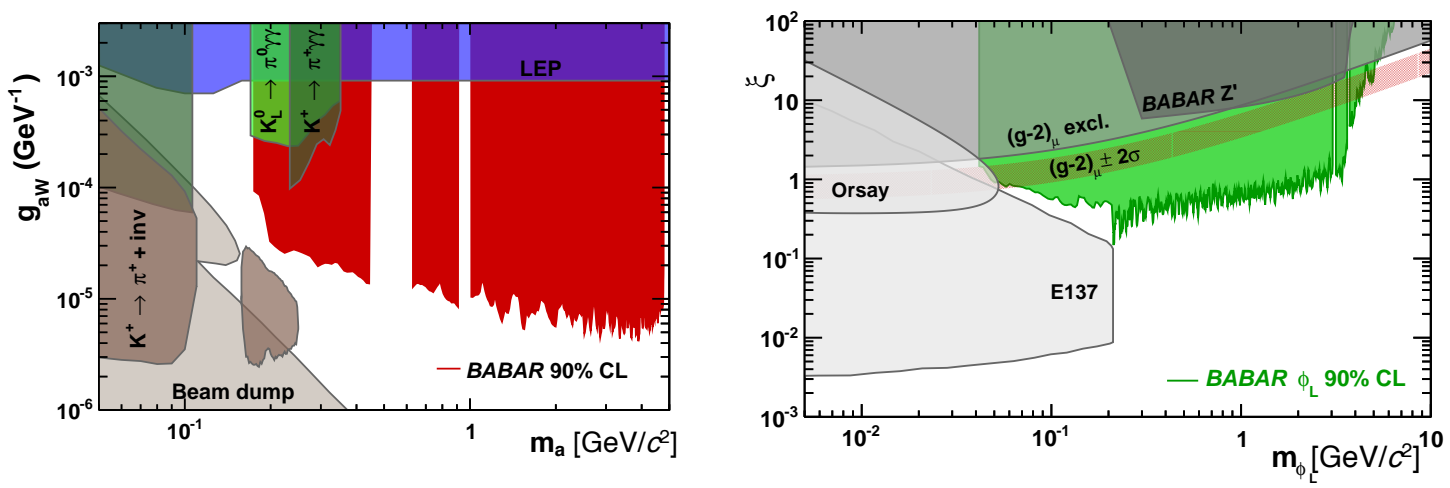

Figure 5: Left: The 90\% CL upper limits on the coupling $g_{\mathrm{aw}}$ as a function of the ALP mass (shaded red region) in comparison to results of other experiments [10]. Right: The 90\% CL upper limits on the coupling parameter $\xi$ as a function of the $\phi_{\mathrm{L}}$ mass (green shaded area), together with existing constraints [11-16].

\section{Search for Leptophilic Dark Matter}

A new light scalar $\phi_{\mathrm{L}}$ is predicted in Beyond the Standard Model models that can mix with the Higgs boson. Experimental constraints disfavor interactions with quarks but interactions with leptons are permitted. Note that a leptophilic scalar could explain the $g-2$ discrepancy [17] and the KOTO experiment excess in $K_{\mathrm{L}} \rightarrow \pi^{0} v \bar{v}$ [11]. The $\phi_{\mathrm{L}}$ couples preferentially to $\tau \mathrm{s}$. BABAR has searched for $e^{+} e^{-} \rightarrow \tau^{+} \tau^{-} \phi_{\mathrm{L}}$. with a subsequent decay $\phi_{\mathrm{L}} \rightarrow e^{+} e^{-}, \mu^{+} \mu^{-}$using an integrated luminosity of $514 \mathrm{fb}^{-1}$ [18]. In this analysis we consider all one-prong $\tau$ decays. We optimize the analysis for each final state and prompt or long-lived $\phi_{\mathrm{L}}$ using individual BDT selections. We extract signals as a function of the $\phi_{\mathrm{L}}$ mass with fits over sliding intervals. The signal efficiency varies between $0.2-26 \%$. We see no signal and extract $90 \% \mathrm{CL}$ upper limits on the cross sections $\sigma\left(e^{+} e^{-} \rightarrow \tau^{+} \tau^{-} \phi_{\mathrm{L}}, \phi_{\mathrm{L}} \rightarrow e^{+} e^{-}, \mu^{+} \mu^{-}\right)$and the $\phi_{\mathrm{L}}$-lepton coupling parameter $\xi$. Figure 5 (right) shows the $90 \% \mathrm{CL}$ upper limits on $\xi$ as a function of $m_{\phi_{\mathrm{L}}}$. The BABAR results provide a significant improvement in a wide mass region over previous bounds. We exclude a large $g-2$ parameter 
space below the $\tau \tau$ threshold.

\section{Conclusion}

Present $e^{+} e^{-}$colliders provide a unique opportunity for testing the concepts of dark sectors via portals to the SM at low energies. BABAR has conducted a rich program to look for dark matter effects and has set many stringent $90 \% \mathrm{CL}$ upper limits on cross sections and couplings. The most recent $B A B A R$ results include world-leading constraints on self-interacting dark matter, axion-like particles and leptophilic dark scalars. BABAR has more analyses ongoing. Eventually, Belle II will improve all our results.

\section{Acknowledgments}

I want to acknoledge the BABAR collaboration for giving this talk and in particular J. McKenna and F. Porter for providing useful comments.

\section{References}

[1] M. Pospelov, A. Ritz, and M. B. Voloshin, Phys. Lett B 662, 53 (2008).

[2] J. Beacham et al., J. Phys. G 47, 010501 (2020).

[3] P. Fayet, Nucl. Phys. B 187, 184 (1981).

[4] B. Holdom, Phys. Lett. B 166, 196 (1986).

[5] H. An et al., Phys. Rev. Lett. 116, 151801 (2016).

[6] F. J. Rogers, H. C. Graboske Jr., and D. J. Harwood, Phys. Rev. A 1, 1577 (1970).

[7] The BABAR Collaboration (J. P. Lees et al.), e-Print: 2106.08529 [hep-ex] (2021).

[8] J. Berkson, Journal of the American Statistical Association 39, 227 (1944).

[9] The BABAR Collaboration, submitted to Phys. Rev. Lett. (2021).

[10] E. Izaguirre, T. Lin and B. Shuve, Phys. Rev. Lett. 118, 111802 (2017).

[11] J. Liu et al., JHEP 04, 197 (2020).

[12] Y. S. Liu, D. McKeen and G. A. Miller, Phys. Rev. Lett. 117 no.10, 101801 (2016).

[13] J. P. Lees et al. [BABAR Collaboration], Phys. Rev. D 94, 011102 (2016).

[14] J. D. Bjorken et al., Phys. Rev. D 38, 3375 (1988).

[15] M. Davier and H. Nguyen, Phys. Lett. B 229, 150 (1989).

[16] B. Batell et al., A. Ritz, Phys. Rev. D 95, 075003 (2017).

[17] The Muon g-2 Collaboration (B. Abi ]et al.), Phys.Rev.Lett. 126-14, 141801 (2021).

[18] The BABAR Collaboration (J. P. Lees et al.), Phys. Rev. Lett 125 -18, 181801 (2020). 